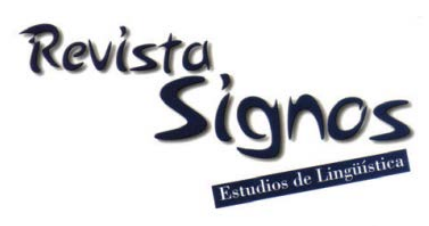

\title{
Escribir historia desde las evidencias. Géneros históricos y sentido histórico en estudiantes de 8vo de Educación Básica
}

\author{
Writing History from the evidence. Historical Recount Genre in \\ elementary 8 th graders
}

\author{
Rodrigo Henríquez \\ PONTIFICIA UNIVERSIDAD CATÓLICA \\ DE CHILE \\ CHILE \\ rodrigo.henriquez@uc.cl
}

\author{
Andrés Carmona \\ INSTITUTO LA SALLE \\ CHILE \\ andres.carmona@lasalle.cl
}

\author{
Alen Quinteros \\ INSTITUTO LA SALLE \\ CHILE \\ alenquinteros@gmail.com
}

Recibido: 22-IV-2015 / Aceptado: 9-VI-2017

\section{Resumen}

Este artículo expone los resultados de una experiencia de investigación realizada el año 2013 con estudiantes de 8vo grado de Educación Básica en un Colegio Particular Subvencionado de Santiago de Chile. El objetivo fue caracterizar los mecanismos histórico-discursivos que los estudiantes utilizaron para responder una pregunta considerando evidencia histórica. El diseño analítico y el metodológico se basan en la noción de género histórico (Coffin, 2006) y el modelo de la VALORACIÓN elaborado desde la Lingüística Sistémica Funcional (LSF) (Coffin, 2006; Martin \& Rose, 2008; Martin, 2009). De la tipología de géneros históricos elaborada y desarrolladas por Coffin (2006) Martin y Rose (2008) se reformuló el género 'recuento histórico' como 'tarea de escritura de recuento histórico'. A partir de la identificación de los recursos lingüísticos recurrentes en los textos se pudo caracterizar 4 etapas (inicio, contextualización, reformulación y cierre) y diferentes fases de realización al interior de cada una de las etapas. Los resultados indican que existen criterios de calidad dados por la cercanía a la evidencia histórica y que se expresan en cada una de las fases al interior de las cuatro etapas caracterizadas en este estudio.

Palabras Clave: Géneros históricos, aprendizaje histórico, escritura histórica, Lingüística Sistémica Funcional. 


\begin{abstract}
The purpose of this paper is to identify and characterize the structure of the historical genre and discursive mechanisms utilized by Chilean $8^{\text {th }}$ graders as evidenced in a written task. This task consists of students responding to a historical question using on historical evidence. Our analytical framework and methodological design are based on the notion of historical genre and Appraisal Framework used in Systemic Functional Linguistics (Coffin 2006; Martin \& Rose, 2008; Martin, 2009). Considering the characterization of historical genres developed by Coffin (2006) and Martin and Rose (2008), the concept of 'historical account' genre is reformulated and defined as a 'historical account writing task.' Our analysis helped identify and characterize four stages to develop the historical account: a) Start, b) Contextualization, c) Reformulation and c) Closing. We unpack each stage into smaller phases. The results show that there exists a set of criteria afforded by the proximity of the response to the historical evidence, which are expressed in the stages and phases characterized in this study. This set of criteria shapes the quality of the response as evidence of students' historical understanding.
\end{abstract}

Key Words: Historical genres, learning history, writing history, Systemic-Functional Linguistics.

\title{
INTRODUCCIÓN
}

El discurso de la historia, al igual que otros discursos académicos, tiene sus propias particularidades, las cuales están principalmente dadas por su tridimensionalidad: narrativa, argumentativa y explicativa (Coffin, 2006). Por su parte, el discurso pedagógico de la historia ha sido estudiado desde diferentes aspectos: desde su aprendizaje (Wineburg, 1991; Peck \& Seixas, 2008); desde el aprendizaje a través de la escritura (Henríquez \& Ruiz, 2014; Monte-Sano, 2011); desde la perspectiva de los significados elaborados en clases (Manghi, 2013), y desde lo que en la tradición anglosajona se denomina Historical Literacy o 'alfabetización histórica' que integra elementos disciplinares, cognitivos y discursivos (Monte-Sano, 2011). En los últimos 20 años no han sido pocos los estudios que han profundizado en el discurso pedagógico de la historia. Sobre este aspecto se han estudiado las estructuras genéricas en textos históricos producidos por escolares (Coffin, 2006), el discurso evaluativo en profesores de historia (Oteíza, 2009; Oteíza, Henríquez \& Pinuer, 2015, entre otros trabajos). Desde teorías del aprendizaje se han descrito cómo funcionan los procesos de razonamiento histórico (Wineburg, 1991). A pesar de estos estudios, aún queda por indagar un ámbito menos conocido para los historiadores y por los estudios del aprendizaje histórico, como lo es la relación entre las etapas de la estructura genérica del recuento histórico y el uso de la evidencia. Esta caracterización permitiría, entre otras cosas, focalizar los esfuerzos educativos para resolver las dificultades del aprendizaje histórico dadas por la distancia existente entre el saber de la experiencia cotidiana y el conocimiento de la disciplina (Halliday \& Martin, 1993). 
En este contexto, la presente investigación tiene como objetivo principal identificar y caracterizar la estructura genérica y los elementos discursivos del género 'recuento histórico' y su relación con la utilización de la evidencia en un corpus de estudiantes chilenos de 8vo básico. Particularmente, indagamos en cómo los estudiantes elaboran la significación histórica en un texto escrito a partir de evidencias. Para el análisis, se utilizó la noción de 'género histórico' (Coffin, 2004, 2006) y el modelo de la VALORACIÓN (Martin \& White, 2005) elaborados dentro de la Lingüística Sistémica Funcional (LSF). Esta noción y este modelo nos permite, entre otras cosas, identificar los recursos lingüísticos con que se elabora la significación histórica.

Debido a la escasez de estudios nacionales que informen sobre el discurso histórico elaborado por los estudiantes -como expresión del aprendizaje histórico-, decidimos levantar nuestros propios datos y analizar cómo los estudiantes de un contexto situado elaboran sentido histórico a través y con el lenguaje académico. De esta forma, se identificaron los recursos lingüísticos más recurrentes de los textos, así como el contexto de producción de los mismos (objetivos y contenidos curriculares, evidencias y preguntas históricas), cuestión que ha sido pasada por alto por una buena parte de la investigación educativa sobre aprendizaje histórico en Chile de estudiantes de Educación Media (Henríquez \& Ruiz, 2014). El principal aporte de este artículo es informar sobre la utilización de herramientas teórico-metodológicas de la LSF para el desarrollo de la alfabetización histórica en contextos de aula a partir de estrategias pedagógicas que utilizan evidencia histórica, preguntas y producción de textos.

\section{Marco teórico}

\section{Aprendizaje histórico y lenguaje disciplinar}

Estudios recientes sobre aprendizaje histórico señalan que una de las metas educativas más complejas es la escritura de textos históricamente argumentados (Coffin, 2004; Monte-Sano, 2010). Para llevar a cabo dichas metas curriculares -como, por ejemplo, establecer interrelaciones entre causas y consecuencias de un hecho o proceso histórico (Mineduc, 2012)-, se hace indispensable utilizar mecanismos discursivos para llevar a cabo dicho propósito. Sobre este panorama, la elaboración de relaciones entre causas y consecuencias es una de las finalidades curriculares más extendidas en la enseñanza de la historia a escala internacional. A través de su enseñanza, entre otras cosas, se busca desarrollar la comprensión y la construcción del sentido histórico (Coffin, 2004; Peck \& Seixas, 2008). Por su parte, los estudios sobre alfabetización histórica han remarcado la relevancia del lenguaje disciplinar como puerta de acceso al aprendizaje y al mundo social. El lenguaje disciplinar y pedagógico de la Historia, así como el de otras disciplinas humanistas y sociales, comparte la peculiaridad de tener un lenguaje especializado con características particulares como la utilización de nominalizaciones. Estas participan en la construcción más abstracta de 
los conceptos y esto, particularmente en la Historia, se traduce en un empaquetamiento o cosificación de los procesos y del tiempo. También propician la tendencia a la objetivación y la existencialización de los eventos, junto con el tratamiento impersonal de las relaciones causales que tienden a realizarse al interior de las cláusulas (Coffin, 2006; Oteíza, 2006, 2009, 2011; Martin \& Rose, 2008; Moyano, 2010; Oteíza \& Pinuer, 2012; Manghi, 2013). Estas cualidades del discurso histórico permiten interpretar y explicar en el texto histórico las relaciones entre agentes, eventos y procesos estableciendo causas y consecuencias entre ellos (Coffin, 2004).

\subsection{Estudios lingüísticos sobre géneros históricos}

Un género es entendido como un proceso social orientado a fines que presenta cierta estructura organizativa (Coffin, 2006; Martin \& Rose, 2008; Martin, 2009). En el caso de la Historia, los géneros históricos escolares han sido caracterizados (Coffin, 2006; Martin \& Rose, 2008) a partir de diferentes registros escolares y géneros. De esta manera, encontramos textos producidos por estudiantes de educación básica y secundaria (Coffin, 2006), libros de textos de Historia (Oteíza, 2009, 2011; Altamirano, Godoy, Manghi \& Soto, 2014) e interacciones de clase (Manghi, 2013; Manghi, Badillo \& Villacura, 2014). A su vez, la producción de textos históricos tiene particularidades que permiten agruparlos en tres familias de géneros: a) géneros narrativos, b) géneros explicativos y c) géneros argumentativos (Coffin, 2006). Una de las características de los géneros narrativos es que relaciona eventos concretos y particulares, inscritos en una temporalidad lineal. En los géneros narrativos no hay aparato interpretativo ni estructuras conceptuales que organicen, conecten y orienten a un conjunto de hechos; más bien hay una sucesión temporal que es el eje que los articula. En este grupo de géneros se reconoce el 'relato autobiográfico' y el 'biográfico', el 'recuento histórico’ y ‘el informe histórico’. Según Coffin (2006), esta familia de géneros es la más recurrente en las aulas de historia australianas donde la autora realizó su estudio. La familia de los géneros 'explicativos' tendría como propósito la explicación de eventos del pasado a través de la examinación de causas y consecuencias integrando una multiplicidad de factores de largo y corto plazo. Componen esta familia los géneros de 'explicación factorial' y 'explicación consecuencial'. Por último, se encuentra la familia de los géneros argumentativos que tienen como propósito utilizar los puntos de vista de la historia para la construcción de una narración con significado histórico. Los géneros que componen esta familia son la 'exposición', ‘discusión’ y ‘desafío’.

Investigaciones recientes que han utilizado las nociones de género histórico en contextos de aula en Chile han contribuido a identificar las características discursivas del aprendizaje histórico y algunos de sus problemas. Básicamente, uno de estos problemas refiere a la dificultad para utilizar argumentos apoyados con la evidencia como garantía para elaborar un nuevo argumento en un texto escrito (Henríquez \& Canelo, 2014; Oteíza, Dalla Porta \& Garrido, 2014). Esta dificultad también es 
frecuente en estudiantes universitarios de historia (Henríquez \& Canelo, 2014; Oteíza et al., 2014). En estos estudios se evidencia que las explicaciones históricas de estudiantes de Licenciatura en Historia se presentan con mayor o menor peso argumentativo y con mayor abstracción dependiendo de los tipos de los recursos lingüísticos utilizados para incluir la perspectiva de las evidencias en la escritura y, así, elaborar la significación histórica. Otras investigaciones han utilizado la noción de 'recuento histórico' para analizar interacciones de clases de Historia (Manghi, 2013; Manghi et al., 2014). De estos estudios destaca la caracterización de los recursos semióticos utilizados en la instrucción y los géneros predominantes: explicaciones de causas y de recuento histórico. Sin embargo, el foco de estos estudios es el discurso del profesor no entrando mayormente en elementos disciplinares tales como los contenidos, los textos utilizados, las fuentes y las preguntas históricas.

Con respecto a la noción del género recuento histórico (Historical Reccount), su objetivo es dar cuenta de la sucesión de eventos de un hecho histórico para establecer la línea de tiempo de la 'gran narrativa'. Es decir, el pasado se construye como una versión objetiva, factual y lógica en la que el autor parece dejar que los eventos 'hablen por sí solos'. Se trata de un género que al registrar hechos y eventos antes y después en una narrativa tiende a explicar y elaborar el sentido histórico (Coffin, 2006). El propósito pedagógico de este género es otorgar al estudiante recursos lingüísticos para la generalización, debido a que no se centra tanto en personas individuales, sino en grupos de personas procesos y hechos. Para Coffin (2004) el recuento histórico posee tres etapas funcionales: a) Orientación (Background), el cual provee de un resumen de los eventos históricos previos que son significativos para el texto; b) Registro de Eventos (Record of events), el cual proporciona un registro de los principales eventos históricos; c) Deducción (deduction), el cual proporciona la significancia histórica de los acontecimientos que se han registrado (Coffin, 2004.) Como indica Coffin (2004, 2006), el recuento histórico es uno de los más utilizados en la enseñanza para la presentación y construcción de las versiones del pasado.

\subsection{Noción de género, nominalizaciones y modelo de la VALORACIÓN}

En la presente investigación utilizamos la noción de género propia de la LSF, la cual entiende este concepto como las opciones recurrentes de elecciones semánticas que se desarrollan en etapas y apuntan a la realización de un propósito social compartido culturalmente (Martin \& Rose, 2008).

Las nominalizaciones son una forma de metáfora gramatical cuya principal característica es la transformación de procesos en cosas. Como consecuencia, se produce la abstracción y el empaquetamiento de la información (Moss, Barletta, Chamorro \& Mizuno, 2013). Aunque la presencia de nominalizaciones indica ciertos grados de abstracción no necesariamente se relaciona con mayores grados de 
elaboración. Aunque, como señalan Coffin (2006) y Moss et al. (2013), las nominalizaciones son propias del lenguaje académico abstracto, su inclusión no necesariamente asegura calidad, pues pueden ser utilizadas sin sentido histórico o sin conexión léxica con la argumentación del texto.

Para la identificación de los recursos lingüísticos más recurrentes utilizados por los estudiantes para elaborar sus respuestas históricas, se utilizaron los subsistemas de actitud y compromiso del modelo de la VALORACIÓN (Martin \& White, 2005) modificado por Oteíza y Pinuer (2012), el cual permite identificar las evaluaciones que los escritores utilizan para negociar las posiciones subjetivas, particularmente en el discurso histórico. A través del sistema de actitud, se pueden identificar los significados que los estudiantes utilizan para evaluar participantes, procesos históricos y eventos y de esta forma describir las valoraciones inscritas y evocadas que expresan la subjetividad en el discurso. A través del sistema de compromiso se identifican los recursos lingüísticos utilizados para construir la evidencialidad y así reconocer la fuente de los significados interpersonales inscritos o evocados en el discurso (Oteíza \& Pinuer, 2012; Oteíza et al., 2014). Estos significados pueden materializarse en orientaciones monoglósicas (sin voces en el texto) y/o heteroglósicas (presencia de más voces en el texto).

\section{Metodología}

\subsection{Corpus}

Los textos corresponden a estudiantes de 8vo básico de dos cursos de un establecimiento particular subvencionado ubicado en la comuna de la Florida en Santiago de Chile. Se seleccionaron 60 textos producidos a partir de una actividad evaluativa desarrollada durante el segundo semestre del año 2013 correspondiente a la unidad temática 'La expansión europea y su expresión geográfica'. El propósito pedagógico de la actividad se determinó a través del Aprendizaje esperado definido por el Ministerio de Educación: Dimensionar geográficamente la expansión de la influencia europea hacia otros continentes a través de procesos de exploración y conquista durante la época Moderna (Mineduc, 2012). El tiempo de implementación fue de 10 horas pedagógicas y consistió básicamente en clases expositivas y el trabajo con un portafolio de 3 mapas correspondientes a diferentes épocas que describían la expansión europea. La producción del texto final, individual, tuvo la finalidad de responder la pregunta histórica: ¿Cómo la evolución del conocimiento geográfico representado en estos documentos refleja la expansión del mundo europeo?

Las fuentes utilizadas fueron tres: Fuente $\mathrm{n}^{\circ} 1$ mapa del siglo XIX en que se representa el mundo tal como fue concebido durante la Edad Media. Aparecen Europa, el Mediterráneo, el Norte de África y Jerusalén. Fuente nº mapa que corresponde a la representación del mundo que hace Portugal en 1502. Este mapa ofrece detalles de la costa americana y de las islas de las Antillas. Al ser de manufactura 
portuguesa, destaca la importancia del Meridiano que representa el tratado de Tordesillas. Además, representa una visión del mundo directamente relacionada con el motivo de los descubrimientos geográficos del siglo XVI: lo económico. Las rutas comerciales se asocian directamente con el conocimiento geográfico, y el avance en la cartografía. 3) Fuente $n^{\circ} 3$ mapa elaborado en Francia en 1700 gracias a desarrollo del conocimiento científico. Presenta información más detallada sobre zonas que no habían sido suficientemente conocidas, pero se dejan en blanco las no exploradas hasta la fecha de elaboración. Los estudiantes tuvieron 4 horas pedagógicas para responder la pregunta. Para efectos de este reporte, se seleccionaron 2 fragmentos representativos de cada una de las fases de realización.

\subsection{Procedimientos}

Para dar cuenta del nivel de abstracción de los escritos, este estudio se focalizó en los sistemas semánticos de actitud y compromiso. Además, para complementar este aspecto, se incorporó la dimensión de construcción de la causalidad y la presencia de nominalizaciones. A su vez, la dimensión de construcción de la causalidad, se caracterizó a través de la identificación de los recursos lingüísticos utilizados para elaborar la causalidad: de forma congruente por medio de conjunciones ('ya que', 'porque', 'gracias al') y de forma incongruentes a través de verbos (refleja, permitió, ayudó) (Tabla 1).

Tabla 1. Sistema de actitud, Sistema de compromiso y dimensiones de Temporalidad y Causalidad.

\begin{tabular}{|c|c|c|c|c|}
\hline $\begin{array}{l}\text { Qué y quiénes } \\
\text { son evaluados/ } \\
\text { representados }\end{array}$ & $\begin{array}{l}\text { Discurso } \\
\text { (completo) }\end{array}$ & $\begin{array}{l}\text { Cómo son } \\
\text { evaluados }\end{array}$ & $\begin{array}{c}\text { Por quiénes son } \\
\text { evaluados } \\
\text { (Evidencialidad) }\end{array}$ & $\begin{array}{c}\text { Temporalidad } \\
\text { (cronológica y simbólica) } \\
\text { Causalidad }\end{array}$ \\
\hline $\begin{array}{l}\text { Actores sociales } \\
\text { Procesos } \\
\text { Eventos } \\
\text { Cosa/entidad no } \\
\text { humana }\end{array}$ & $\begin{array}{l}\text { Discurso } \\
\text { analizado }\end{array}$ & $\begin{array}{l}\text { Sistema de } \\
\text { actitud } \\
\text { (afecto, juicio } \\
\text { apreciación) }\end{array}$ & $\begin{array}{l}\text { Sistema de } \\
\text { compromiso } \\
\text { Heteroglosia } \\
\text { Monoglosia }\end{array}$ & $\begin{array}{l}\text { Evidencias de la } \\
\text { construcción de las } \\
\text { explicaciones históricas } \\
\text { desde las dimensiones } \\
\text { temporales y causales }\end{array}$ \\
\hline
\end{tabular}

La identificación de las etapas del género se efectuó al segmentar los textos por la función que cumplían los recursos lingüísticos para el logro de la significación histórica (Coffin, 2004, 2006). En este contexto, entendemos por significación histórica, la plausibilidad que se le otorga al discurso histórico en función de un propósito. La función se estableció al identificar cuatro aspectos: 1) El qué y quiénes son evaluados (actores sociales, procesos, eventos, cosa/entidad no humana); 2) Por cómo son evaluados (sistema de actitud); 3) Por quiénes son evaluados (sistema de compromiso); 4) Por la identificación de las relaciones causa-efecto (causalidad). Sobre este aspecto, las relaciones de causa efecto que se realizan por medio de verbos causales establecen una congruencia entre dos nominalizaciones. Esta forma es un 
rasgo característico del lenguaje científico, a diferencia de la relación causa-efecto establecida por las conjunciones que vinculan principalmente procesos materiales.

Para la caracterización de las nominalizaciones se utilizaron los criterios planteados por Chamorro y Moss (2011) y Moss et al. (2013). Para identificar el uso sin sentido o 'estratégico' de nominalizaciones, se estableció un grado de correspondencia con los planteamientos nucleares de evidencia histórica (Henríquez \& Canelo, 2014).

A continuación, en la Tabla 2 presentamos la leyenda que utilizamos para etiquetar los textos:

Tabla 2. Marcas para identificar los recursos lingüísticos presentes en los textos analizados.

\begin{tabular}{|l|l|}
\hline versales minúscula: sistema semántico & Subsistema de Apreciación de procesos y eventos \\
Negrita y subrayado: valoraciones inscritas & sociales: $\uparrow \downarrow$ poder; conflictividad $+/-$ \\
\hline Negrita y cursiva: valoraciones evocadas & Negrita: procesos \\
Valoración positiva $(+)$ valoración negativa $(-)$ & Doble subrayado: nominalización \\
\hline
\end{tabular}

\section{Resultados}

\subsection{Etapa inicio}

La principal característica de esta etapa está dada por la adhesión al propósito de la tarea asignada en la pregunta ¿Cómo la evolución del conocimiento geográfico representado en estos documentos refleja la expansión del mundo europeo? En la mayoría de los textos se aprecia que la valoración se realiza a través del sistema de actitud, subsistema de Apreciación, ya que lo evaluado son procesos (conocimiento geográfico y expansión europea). Mediante la caracterización de las valoraciones inscritas se pudo establecer el grado de adhesión a la pregunta planteada en la tarea y la calidad de dicha adhesión. En esta etapa se definieron 3 fases de realización: a) ausencia de propósito, b) inversión de propósito y c) reformulación de la pregunta. En la Fase ‘ausencia de propósito' hay diferentes grados de adhesión a este. En el ejemplo 1 se fuerza la relación con la pregunta a partir del verbo 'encajar'. En esta fase, la finalidad de los textos no está relacionado con el propósito de la pregunta. Si bien en las respuestas se aprecia que son los textos los evaluados (ejemplo 1 'El texto'), el propósito no se ajusta a la tarea demandada. A pesar de esto, los estudiantes intentan acercarse al objetivo propuesto a través de la nominalización 'conocimiento europeo' (ejemplo 1) pero sin lograr conducir su respuesta a la tarea asignada.

\footnotetext{
Ejemplo 1. El texto nos ayuda a encajar la evolución del conocimiento europeo sobre la geografía y su expansión. (ID 24)

Qué y quienes son evaluados: el texto; el conocimiento europeo

+ apreciación Valuación (nos ayuda). + apreciación Valuación. ComposiciónComplejidad (evolución)
} 
En la Fase 'inversión del propósito', la principal característica es la inversión de la relación causa (expansión geográfica) con la consecuencia (el conocimiento geográfico) que se aprecia en el ejemplo 2. Al igual que en la fase anterior, se utilizan nominalizaciones para referirse al foco de la pregunta, pero se invierte la relación causa-efecto. Nuevamente, lo evaluado por los estudiantes son las nominalizaciones conocimiento geográfico y expansión del mundo europeo.

Ejemplo 2. La evolución de conocimiento geográfico permite la expansión del mundo. (ID 13)

Qué y quiénes son evaluados: la evolución del conocimiento geográfico + apreciación Valuación $\uparrow$ Poder (permite)

Nominalizaciones: el conocimiento geográfico; la expansión del mundo

En la Fase 'Reformulación de la pregunta' se observa una gradualidad entre la reformulación literal de la pregunta a la reformulación de los planteamientos nucleares del propósito como es el caso del ejemplo 3 y la utilización del verbo 'refleja'.

Ejemplo 3. El objetivo de realizar este texto es explicar como la evolución del conocimiento geográfico refleja la expansión del mundo Europeo. (ID 11)

Qué y quiénes son evaluados: El objetivo de realizar este texto

+ apreciación Valuación (explicar)

Nominalizaciones: el conocimiento geográfico; la expansión del mundo

En suma, las fases de la etapa 'inicio' varían en los textos entre la ausencia del propósito a la fase de mayor complejidad dada por la reformulación de la pregunta. Aunque en todas las fases los estudiantes utilizan nominalizaciones lo que podría indicar ciertos grados de abstracción, estas solo indican la comprensión del tópico tratado, mas no la comprensión del propósito de la tarea de escritura demandada en clases.

\subsection{Etapa contexto}

En esta etapa, los estudiantes presentan elementos de contexto temporal y espacial que permiten dar una panorámica general del proceso, o de 'gran narrativa' (Coffin, 2006). El rasgo fundamental es la utilización de información presente en la evidencia. Por ello, se distinguen dos fases: a) de 'Ausencia de evidencia', en que se presentan contenidos de las evidencias, pero de forma genérica sin estar orientadas a la tarea demandada y b) 'Fase de inclusión de tiempo y espacio', donde los estudiantes incorporan referencias espaciales y temporales presentes en la evidencia. En la primera fase los estudiantes presentan referencias espaciales y temporales que enmarcan el proceso de expansión europea. Estas referencias están presentes en las imágenes de la guía y fueron leídas con los alumnos en al menos 4 horas de clases. En esta fase, la variación se da entre la no inclusión de dichas referencias tempo-espaciales en el texto. 
Como se aprecia en el ejemplo 4, la referencia espacial es genérica (Europa) y el proceso que lleva a cabo expresado en la nominalización 'expansión del mundo europeo' no aparece en las evidencias como el antecedente que el estudiante menciona (el bloqueo de Constantinopla).

Ejemplo 4. Entre la edad media hacia adelante ocurrieron muchos procesos y uno de estos procesos fue la expansión del mundo europeo porque buscaban nuevas rutas para el comercio por el bloqueo en Constantinopla. (ID10)

Qué y quiénes son evaluados: Europeos [expansión del mundo europeo] + juicio Estima Social. Tenacidad [buscaban nuevas rutas por el bloqueo]

Monoglosia, cláusulas afirmativas.

Nominalizaciones: expansión del mundo europeo, bloqueo en Constantinopla.

En la Fase de 'inclusión de tiempo y/o espacio', se aprecia un mayor grado de complejidad dado por la inclusión de referencias temporales presentes en la evidencia, pero con escasa utilización de conceptualizaciones espaciales. Este aspecto se aprecia en el ejemplo 5, en que solo se mencionan los siglos en que se elaboraron los mapas. Las referencias temporales están ordenadas de forma cronológica y secuencial como se aprecia en las marcas de temporalidad de los siglos (XI-XVIII; XII- XIII, XVI y XVIII).

Ejemplo 5. Este proceso de evolución fue muy largo y comprendió los siglos XIXVIII. (ID24)

Qué y quiénes son evaluados: Este proceso de evolución

+/- apreciación. Valuación [largo, no se sabe si es valoración positiva o negativa de la duración del proceso]

Monoglosia, cláusula afirmativa.

Nominalizaciones: Este proceso de evolución

\subsection{Etapa reformulación}

La principal característica de esta etapa es que los estudiantes proporcionan la garantía que permite relacionar la causa (la expansión geográfica) con la consecuencia (el conocimiento geográfico). De esta manera, tanto la causa como la consecuencia aparecen nominalizados mediante verbos causales. Por su parte, la garantía es la presencia explícita de conceptos y datos dados por la evidencia y detectados gracias al sistema de compromiso. A través de esto, los estudiantes pueden relacionar los conceptos presentes en nominalizaciones con los efectos de los procesos históricos. Se identificaron 4 fases: a) Fase de 'Ausencia de evidencia'; b) Fase de 'Crónica factual'; c) Fase de 'Secuencia cronológica' y d) Fase de 'Perspectiva sobre la evidencia'.

La primera fase oscila entre la 'Ausencia de evidencia' y la utilización de solo una parte de la información presentada en uno de los mapas como el desarrollo 
tecnológico junto a otras informaciones que no aparecen en las evidencias. En el ejemplo 6, la causa para la evolución del conocimiento geográfico se produjo por la nominalización 'la curiosidad científica'. Otro elemento que aparece como causa en la misma respuesta son fenómenos que no estaban en las evidencias (el mundo era plano y habían monstruos). La construcción de la agencia se realiza desde lo que los personajes quieren ('los hombres y mujeres modernos querían vivir grandes aventuras'). Esto lo caracteriza la evaluación desde el sistema de juicio y el subsistema de Estima Social junto al de Afecto. En cuanto a la incorporación de otras voces, el ejemplo 6 presenta una orientación heteroglósica por intravocalización de expansión dialógica en relación a los factores ('una serie de...') que explican la expansión geográfica. Dado que dichos factores están presentes en las evidencias, su inclusión en el texto da cuenta de una reformulación de la evidencia y, por tanto, de heteroglosia. Sin embargo, esta 'apertura' dialógica es limitada por la utilización estratégica de nominalizaciones (la evolución del conocimiento geográfico y la curiosidad científica) que se sustentan en información no respaldada. Junto a ello, la respuesta se traslada de valoraciones de apreciación a evaluaciones de juicio y afecto que indican que la orientación histórica se realiza desde la agencia emocional y desde sus estados mentales ('pensaban', 'quería vivir') de los personajes históricos ('hombres y mujeres modernos').

Ejemplo 6. La evolución del conocimiento geográfico se devia a una serie de entre ellos está la curiosidad científica por conocer y poder comprender el mundo y que ellos pensaban que el mundo era plano en sus esquinas habia un vacio lleno de monstruos entre otros está que al hombre le salía ese espíritu de explorador que caracterizo a hombres y mujeres modernos quienes querían vivir grandes aventuras lograr fama riqueza y poder ellos anteriormente solo hacían mapas de lo que veian y lograron explorar más alla de su vicion. (ID15)

Qué y quiénes son evaluados: evolución del conocimiento geográfico, una serie de factores, Ellos, al hombre/hombres y mujeres modernos

+ apreciación Composición. Complejidad. [se debe a una serie de ]; + apreciación . Valuación [son causa de la evolución del conocimiento geográfico] curiosidad, conocer y comprender; - juicio. Estima social. Capacidad, Tenacidad (pensaban que el mundo era plano); + juicio. Estima Social. Tenacidad (espiritu explorador); +AFECTO. Querian vivir grandes aventuras y lograr fama, riqueza y poder. + juicio . Estima Social. Capacidad/Tenacidad [a pesar de que solo hacían mapas de lo que veían, lograron explorar más allá de su visión] Heteroglosia. Intra-vocalización. Expansión dialógica. Consideración. Una serie de, entre ellos. Entre otros, está

Nominalizaciones: la evolución del conocimiento geográfico, la curiosidad científica, los hombres y las mujeres modernos. 
La segunda fase de esta etapa, la 'Crónica factual', varía entre relatos sin referencias temporales a otros con una secuencia organizada cronológicamente que no se refiere al efecto de la expansión geográfica que es el conocimiento del mundo. Esto se aprecia en el ejemplo 7 en que la crónica factual se elabora uniendo hechos de forma congruente a través de conjunciones ('gracias al') y de forma incongruente a través de verbos en pasado y presente ('hacían', 'crear', 'haber', 'tener'). La crónica se realiza a través de la unión clausual conectadas por un punto seguido y no por una marca textual que indique la presencia de una cadena causal. La evaluación de los participantes (los europeos y conocimiento geográfico) se realiza predominantemente desde los subistemas de apreciación - Valuación y de juicio - Estima Social lo que indica ciertos grados de abstracción. A su vez, hay escasas menciones a la evidencia y en su inclusión en el texto predomina la orientación heteroglósica por expansión dialógica.

Ejemplo 7. Tenían mapas de europa que los hacían al ojo parándose en distintos lugares y navegando por las costas para crear una referencia de cómo era Europa. Ese era su conocimiento geográfico en el siglo XI. En el siglo XVI hubieron descubrimientos geográficos como saber que el mundo era mucho más grande y era circular como también tener nuevas rutas comerciales con diferentes continentes del mundo gracias al avance de la cartografia. En el siglo XVIII el mundo ya estaba recorrido en su gran mayoría gracias al conocimiento científico este se ve más preciso y el conocimiento geográfico es un fiel reflejo como las ciencias se hacen $\underline{\text { mas }} \underline{\text { rigurosas (ID29) }}$

Qué y quiénes son evaluados: los europeos (evocado), descubrimientos geográficos, el mundo, conocimiento científico, conocimiento geográfico, ciencias.

- juicio-Estima Social Capacidad (no ocupaban la razón); + juicio- Estima Social Capacidad (aún así conocían); - juicio-Estima social Capacidad (aunque pensaban que era África) ; + apreciación- Valuación [está a centro]; Reacción Impacto; + juicio- Estima Social Capacidad (los hacian al ojo) Tenacidad (crear una nueva referencia); +/- juicioCapacidad [estado de su conocimiento];

+ apreciación- Valuación, [los descubrimientos fueron positivos, nuevas rutas comerciales, avance de cartografía]

+/- apreciación-Reacción Impacto (estaba recorrido, se ve más preciso); + apreciación Valuación $\uparrow$ Poder [gracias a éste el mundo se ve más preciso]; + apreciaciónValuación (fiel reflejo) ; + apreciación -Composición Complejidad, (se hacen más rigurosas)

Heteroglosia, Intra-vocalización Expansión dialógica Consideración Bueno, como Evidencialidad: se demuestran

Monoglosia, cláusula afirmativa 'Tenian mapas de europa que los hacían al ojo parándose en distintos lugares y navegando por las costas para crear una referencia de cómo era Europa', cláusula afirmativa: 'Ese era su conocimiento geográfico en el siglo XI' 
Expresión cronológica del tiempo: verbos en tiempo pasado y presente, siglo XVI, nuevas. Verbos en tiempo pasado y presente, siglo XVIII, Verbos en tiempo pasado y presente.

Expresión congruente de causalidad: gracias al

Nominalizaciones: conocimiento geográfico, conocimiento científico, descubrimienos geográficos

En la tercera fase denominada 'Crónica orientada a consecuencias' se aprecia un mayor grado de construcción de la cadena causal donde los textos relacionan la causa histórica (expansión geográfica), y la secuencia de hechos con los efectos producidos (conocimiento geográfico) utilizando la evidencia presentada. En el ejemplo 8 se establece la secuencia de mapas y al final aparece la consecuencia en el conocimiento científico. En el ejemplo 8 también se establece la relación causa-consecuencia a través de un relato cronológico que pone énfasis en los efectos de la expansión geográfica utilizando para ello la evidencia de los mapas. Se aprecia un mayor grado de abstracción por las evaluaciones realizadas desde el subsistema de apreciación y con mayor perspectiva de la evidencia por la orientación heteroglósica del texto.

Ejemplo 8. La primera fuente (mundo según los ingleses, siglo XI) nos muestra la visión que tenían los europeos de la edad media. Debido a las características del mundo medieval, este mapa presenta un mundo teocéntrico. La segunda fuente (cantino, Portugal, siglo XVI) este mapa presenta una visión de mundo directamente relacionada con el motivo de los descubrimientos geográficos, en la cartografía por la aparición de los puntos cardinales. La tercera fuente (mapamundi de Deslile, siglo XVIII) el conocimiento científico se hace cada vez más preciso, y en los conocimientos geográficos aparecen los polos. (ID 24)

Qué y quiénes son evaluados: primera fuente, europeos, mapa, la fuente/este mapa, visión de mundo, conocimiento científico.

+ apreciación- Valuación Nos muestra; +/-JUICIO Capacidad (visión que tenían);

+ apreciación- Valuación (presenta una visión de mundo) ; + apreciación- Valuación (directamente relacionada)

+ apreciación- Composición Complejidad (se hace más preciso) ; + apreciaciónComposición Complejidad (aparecen los polos)

+ apreciación-Composición Complejidad (se hace más preciso)

+ apreciación-Composición Complejidad (aparecen los polos)

Heteroglosia Extra-vocalización Asimilación Nos muestra. Intra-vocalización Expansión dialógica, Consideración Tercera fuente, Segunda fuente, presenta

Evidencialidad: primera fuente

Nominalizaciones: mundo medieval; mundo teocéntrico, conocimiento geográfico

La última fase corresponde a la denominada 'Narración basada en evidencias', en esta el relato se construye en la relación causa y efecto utilizando de forma simultánea 
referencias temporales y espaciales señaladas en la evidencia. En el ejemplo 9, se aprecia como la nominalización 'el conocimiento geográfico' mencionada en los anteriores casos, es sustentada con información presente en la evidencia y evaluada predominantemente desde el subsistema de apreciación, que indica mayores grados de abstracción. La reformulación de la información de las evidencias se realiza con recursos lingüísticos que indican la orientación heteroglósica por intravocalización. Asimismo, la construcción de la temporalidad se realiza con marcas inscritas y la causalidad utiliza expresiones congruentes de causalidad por conjunciones e incongruentes por verbos en pasado. Sobre este aspecto, existen dos aspectos que favorecen la calidad histórica de esta respuesta: por un lado, la construcción de una narración utilizando la evidencia y por otro lado, la perspectiva que el autor tiene sobre ella.

Ejemplo 9. El conocimiento geográfico se vio presentado en los mapas el hombre pudo desarrollar mas espacios geográficos y esto fue fundamental en el comercio, aunque no altiro, porque al principio en el siglo XI el primer mapa no se hizo no estaba todo desarrollado no tenia definido que una europa y asia entonces no les ayudaba mucho. Pero a medida del tiempo el conocimiento geográfico fue evolucionando porque iban descubriendo mas continentes e iban haciendo mas mapas (desarrollados) se iba expandiendo europa porque iban conociendo mas y lo traspasaban a los mapas al igual que los otros continentes, ya que en el otro lado ya estaba bien desarrollado los conocimientos geográficos de el hombre, ya que descubrió america y estaban definidos los continentes y océanos y fue también evolucionando el conocimiento científico, como el origen da las cartografias, esto ayudó a la navegacion de los europeos a descubrir más continentes y eso fue lo más importante porque lo que descubrían lo pasaban a los mapas y a medid que se hacia eso se iba expandiendo europa y el mundo. (ID 28)

Qué y quiénes son evaluados: El hombre, desarrollo de más espacios geográficos, Primer mapa, Conocimiento geográfico, Los conocimientos geográficos, Descubrimiento de los europeos, El paso de los descubrimientos a los mapas.

+ juicio- Estima Social Capacidad (Pudo desarrollar).

+ apreciación-Valuación (fue fundamental en el comercio)

- apreciación- Valuación $\downarrow$ Poder (no les ayudaba mucho)

+ /- apreciación- Valuación (fue evolucionando)

+ juicio-Estima Social

Capacidad/Tenacidad (iban descubriendo, iban haciendo, iban conociendo)

+ apreciación-Composición Complejidad (estaba bien desarrollado)-

+ juicio- Estima Social Capacidad (descubrió América)

+ apreciación- Composición Complejidad (fue evolucionando) Valuación (esto ayudó)

+ apreciación- Valuación (lo más importante)

+ apreciación- Valuación (a medida que se hacía eso se iba expandiendo Europa) 
Heteroglosia Intra-vocalización, Expansión dialógica, consideración (Esto fue fundamental, entonces). Intra-vocalización, Contracción dialógica Negación Aunque (...) no se hizo, Pero. Intra-vocalización, Expansión dialógica, Consideración, También, al igual que, como

Expresión cronológica del tiempo: Altiro, al principio en el siglo XI, primer. Verbos en tiempo pasado, a medida del tiempo, evolucionando (x2).

Expresión congruente de causalidad: Porque (x3), ya que (x2).

Actores sociales agentes: El hombre los hombres.

Expresión incongruente de causalidad: Ayudó

Nominalizaciones: Conocimiento geográfico, conocimiento científico.

\subsection{Etapa cierre}

La última etapa de 'cierre' se caracteriza por dar orientación final al recuento histórico e intentar responder la pregunta de la actividad. En ella se presentan 3 fases de realización según la complejidad de su elaboración. La primera fase denominada 'Orientación por avance del tiempo' se caracteriza por describir el efecto en función del avance del tiempo. En el ejemplo 10, el estudiante reformula la pregunta a partir de la construcción congruente de la causalidad (ya que) en función del avance del tiempo. Esto se evalúa predominantemente desde el subsistema de apreciación.

Ejemplo 10. [El conocimiento geográfico refleja la expansión del mundo europeo ya que cada mapa es más avanzado que el anterior y la mayoría de las tierras representadas estaban bajo el control de algún país europeo, porque no SOLO vinieron a descubrit, sino a conquistar, hacerse más poderosos, y conocer más] (ID1)

Qué y quiénes son evaluados: conocimiento geográfico, cada mapa, país europeo.

+ apreciación- Composición Complejidad (refleja la expansión)

+ apreciación-Valuación, Composición, Complejidad (Más avanzado)

+ apreciación- $\uparrow$ Poder [controlan la mayoría de las tierras]

+ juicio- Estima Social Capacidad/Tenacidad (vinieron a descubrir, conquistar, hacerse más poderosos, conocer más)

Heteroglosia Intra-vocalización Expansión dialógica Consideración Cada, el anterior, mayoría. Intra-vocalización Contracción dialógica Negación no sólo, sino

Expresión cronológica de temporalidad: anterior. Verbos en tiempo presente y pasado.

Expresiones congruentes de causalidad: porque, ya que.

Expresión incongruente de causalidad: refleja [expansión del mundo europeo es causa del conocimiento geográfico]

Actores sociales colectivos agentes: país europeo, 'europeos'.

Nominalizaciones: el conocimiento geográfico, la expansión del mundo europeo 
La segunda fase, denominada 'orientación por interpretación', el efecto histórico es interpretado a partir de expresiones congruentes ('ya que') e incongruentes de la causalidad ('permitió) que relaciona la expansión europea con las mejoras en los mapamundi (ejemplo 11). Así 'la expansión europea’ tiene una orientación histórica ya que es explicada por informaciones presentes en la evidencia. Se observa que la valoración principalmente realizada a partir del subsistema de apreciación que indica un mayor grado de abstracción.

Ejemplo 11. El conocimiento permitió la expansión del mundo europeo, ya que el conocimiento se tradujo en mejores representaciones del mapamundi el cual permitió la mejor navegación y ubicación en el planeta para la expansión europea (ID24)

Qué y quiénes son evaluados: el conocimiento, representaciones del mapamundi.

+ apreciación- Valuación $\uparrow$ Poder (permitió la expansión)

+ apreciación- Valuación (mejores)

+ apreciación- Valuación (permitió la mejo

Monoglosia Cláusula afirmativa

Expresión congruente de causalidad: ya que.

Expresión incongruente de causalidad: permitió (x2)

Expresión cronológica de temporalidad: verbos en tiempo presente y pasado.

Nominalizaciones: la expansión del mundo europeo.

En la última fase 'Síntesis con narración y explicación' (ejemplo 12) se sintetizan los planteamientos nucleares de la evidencia a partir de la nominalización 'la expansión' con el efecto 'el conocimiento geográfico' utilizando para ello la garantía que le otorga la evidencia $n^{\circ} 3$. En la respuesta, el estudiante, junto con hacerse cargo de la pregunta, establece un nexo argumentativo basado en la evidencia gracias a expresiones congruentes e incongruentes de la causalidad. Aunque la valoración se realiza desde los subsistemas de juicio y apreciación, la respuesta adquiere perspectiva sobre la evidencia a través de la garantía que otorga la evidencia ('Un ejemplo es la fuente $\left.n^{\circ} 3^{\prime}\right)$.

Ejemplo 12. Y finalmente la evolución del conocimiento geográfico influye directamente con la expansión, ya que ellos al viajar y conocer nuevas tierras $\underline{\text { su }}$ conocimiento aumentaba cada vez más y por eso lo podían representar mejor. Un ejemplo es la fuente $\mathrm{n}^{\circ} 3$ ya que, por sus viajes recopilaron información y la pudieron representar de mejor manera. (ID 1)

Qué y quiénes son evaluados: evolución del conocimiento geográfico, ellos.

+ apreciación-Reacción Impacto (influye directamente)

+ juicio- Estima Social Capacidad/Tenacidad (conocimiento aumentaba cada vez, más, representar mejor)

+ juicio-Estima Social Capacidad (pudieron representar de mejor manera)

Monoglosia, cláusula afirmativa. 
Heteroglosia, Intra-vocalización Expansión dialógica Consideración Un ejemplo

Expresión cronológica del tiempo: evolución, Verbos en tiempo presente y pasado.

Expresión congruente de causalidad: por eso, ya que.

Expresión incongruente de causalidad: influye

Actores sociales colectivos agentes: ellos

Nominalizaciones. La evolución del conocimiento geográfico, la expansión

Un aspecto que llama la atención de esta etapa: la presencia de procesos materiales (descubrir, hacerse, viajar, conocer, representar) con procesos mentales que enlazan nominalizaciones de forma incongruente. La presencia de nominalizaciones con procesos que podrían ser nominalizados, da cuenta de que los estudiantes comienzan a probar maneras de elaborar la abstracción en la etapa de cierre, asociada a las conclusiones y a tesis más generales sobre la pregunta que guió la tarea de escritura. A continuación, en la Figura 1 presentamos de forma esquemática las etapas y fases del género 'recuento histórico' analizado en la presente investigación:

\section{CONCLUSIONES}

En concordancia con los estudios que analizan los procesos de alfabetización histórica en textos escritos de estudiantes de nivel secundario en Historia (MonteSano, 2010; Henríquez \& Ruiz, 2014), este estudio se propuso explorar el desarrollo de los 'géneros históricos' a partir de la propuesta desarrollada por Coffin $(2004,2006)$ y Martin y Rose (2008) con textos elaborados por estudiantes de escuelas chilenas de Educación Básica. Uno de los logros de esta investigación exploratoria fue identificar cuatro etapas y diferentes fases del género 'recuento histórico': inicio, contextualización, reformulación y cierre. Las características de cada una de las etapas tienen un rango que varía entre menos y más abstracción, y entre menos y más utilización de la evidencia histórica proporcionada a los estudiantes. La caracterización de 4 etapas se estableció a partir de la identificación de algunos rasgos lingüísticos más recursivos de las respuestas de los estudiantes. A partir de esto, se definieron al interior de las etapas diferentes fases de concreción (Figura 1). Los criterios para definir la calidad de las fases de concreción se establecieron tanto en las etapas 'inicio' y 'cierre' por la adhesión del texto al propósito de la pregunta e instrucción. Para las etapas de 'contexto' y 'reformulación' se establecieron los criterios de cercanía a la evidencia $(+\mathrm{o}-)$ y de abstracción (+ o -). La cercanía a la evidencia es la calidad de la reformulación de los contenidos nucleares de la evidencia dada a los estudiantes. Esto se pudo establecer a través del Sistema de compromiso y de la construcción de la heteroglosia por extravocalización. Esto permitió evaluar la calidad de las nominalizaciones con la que los estudiantes construyeron la significación histórica. A su vez, la caracterización de la abstracción se realizó a través de la identificación de las valoraciones del sistema de actitud, y del subsistema apreciación que valora entidades no humanas, como lo son las nominalizaciones (Chamorro \& Moss, 2011). 
En síntesis, el resultado más relevante fue la elaboración de criterios que permiten caracterizar el desplazamiento de las fases de menor complejidad a mayor complejidad y la inclusión de información presente (personajes, fechas, conceptos, procesos) en la evidencia. Este último punto, detectado por Oteíza et al. (2014) es un aspecto a seguir explorando para mejorar eficacia de la evaluación del lenguaje histórico escolar. Además, estos resultados expanden la caracterización de géneros históricos en educación superior elaborado por Henríquez y Canelo (2014) y es complementario de los resultados informados por Manghi (2013) y Manghi et al. (2014) para Educación Media dado que incorpora cuestiones como la inclusión de la evidencia en la construcción de argumentos en textos escritos. A diferencia de lo planteado por Coffin $(2004,2006)$ que caracteriza el género escolar 'recuento histórico' en tres etapas (orientación, registro de eventos y deducción), nuestro estudio detectó cuatro etapas articuladas en función del propósito de la tarea de escritura (pregunta de la guía) y especificidades diferenciadas de las fases de realización.

Otro resultado relevante fue la caracterización de la etapa 'inicio' y los grados de adhesión que los autores de los textos establecieron con el propósito de la tarea de escritura. Aunque los autores intentaron acercase a la tarea demandada a través del uso de nominalizaciones como 'el conocimiento geográfico' y 'la expansión del mundo europeo' estas no aseguraron el logro de una respuesta considerada de calidad (que indicara el propósito del texto). Un ejemplo de ello fue la inversión de la causa y el efecto al interior de esta etapa. Lo anterior nos lleva a sospechar que, si bien el uso de nominalizaciones es una marca de un lenguaje más abstracto y que logra empaquetar información, su uso no asegura por si misma la calidad y complejidad del texto. Otro aspecto relevante es la construcción de la agencia. Esta transita desde el sistema de juicio al sistema de apreciación (por ejemplo en el caso del actor social 'los europeos') desde el sistema de juicio la orientación histórica se realiza desde la agencia emocional y desde sus estados mentales ('pensaban', 'quería vivir') de los personajes históricos ('hombres y mujeres modernos'). En la etapa de cierre, la construcción de la agencia se produce desde procesos materiales que pueden ser nominalizaciones. Esto daría cuenta de una apropiación gradual del lenguaje abstracto de la historia. La caracterización los recursos lingüisticos con que los estudiantes de 8vo básico elaboraron el 'recuento histórico' proporciona evidencia consistente sobre la adquisición del pensamiento histórico a través de sus huellas en el lenguaje académico disciplinar. Además, nos permite clarificar las oportunidades de aprendizaje y las prácticas de enseñanza que favorecen su desarrollo (formulación de preguntas históricas, prácticas de lectura de evidencia, prácticas de escritura histórica, retroalimentación). Junto con lo anterior, existen aspectos por donde seguir explorando y que no quedan del todo identificados en este estudio, tal como el papel que juegan las preguntas como modeladoras de las tareas de escritura. Sin embargo esto, la identificación de lo que aquí se ha denominado recursos histórico-discursivos del género 'recuento histórico' nos entrega luces sobre cómo modelar 
pedagógicamente las prácticas de lectura y escritura histórica con estudiantes de educación Básica y Media.

\section{REFERENCIAS BIBLIOGRÁFICAS}

Altamirano, P., Godoy, G., Manghi, D. \& Soto, G. (2014). Analizando los textos escolares de Historia, Geografía y Ciencias Sociales: La configuración multimodal de los pueblos originarios. Estudios Pedagógicos, 40(1), 263-280.

Coffin, C. (2004). Learning to write history: The role of causality. Written communication, 21(3), 261-289.

Coffin, C. (2006). Historical discourse: The language of time cause and evaluation. Londres: Continuum.

Chamorro, D. \& Moss, G. (2011). La pedagogía de la simplificación: El estudio de la historia por medio de la pista y pesca. En T. Oteíza \& D. Pinto (Eds.), En (re)construcción: Discurso, identidad y nación en los manuales escolares de historia y de ciencias sociales (pp. 11-17). Santiago: Editorial Cuarto Propio.

Halliday, M. \& Martin, J. (1993). Writing science: Literacy and discursive power. Londres: Falmer.

Henríquez, R. \& Canelo, V. (2014). Géneros históricos y construcción de la significación histórica en estudiantes de Licenciatura en Historia. Onomazein. Número Especial IX ALSFAL, 138-160.

Henríquez, R. \& Ruiz, M. (2014). Chilean students learn to think historically: Construction of historical causation through the use of evidence in writing. Linguistics and Education, 25(1), 145-157.

Manghi, D. (2013). Géneros en la enseñanza escolar: Configuraciones de significado en clases de historia y biología desde una perspectiva multimodal. Revista Signos. Estudios de Lingüistica, 46(82), 236-247.

Manghi, D., Badillo, C. \& Villacura, P. (2014). Alfabetización semiótica en clases de historia, estrategias de mediación desde un enfoque multimodal. Perfiles Educativos, XXXVI, 146, 63-79.

Martin, J. (2009). Genre and language learning: A social semiotic perspective. Linguistics and Education, 20(1), 10-21.

Martin, J. \& White, P. (2005). The language of evaluation. Appraisal in English. Nueva York: Palgrave Macmillan.

Martin, J. \& Rose, D. (2008). Genre relations: Mapping culture. Londres: Equinox. 
Ministerio de Educación de Chile (2012). Historia, Geografía y Ciencias Sociales, Programa de Estudio para Octavo Año Básico, Unidad de Currículum y Evaluación. Santiago.

Ministerio de Educación de Chile (2012). Bases Curriculares de la Educación Básica. Historia y Geografia y Ciencias Sociales. Santiago Gobierno de Chile. Ministerio de Educación.

Monte-Sano, C. (2010). Disciplinary literacy in history: An exploration of the historical nature of adolescents' writing. Journal of the Learning Sciences, 19(4), 539-568.

Monte-Sano, C. (2011). Beyond reading comprehension and summary: Learning to read and write by focusing on evidence, perspective, and interpretation. Curriculum Inquiry, 41(2), 212-249.

Moyano, E. (2010). Aportes del análisis de género y discurso a los procesos de enseñanza y aprendizaje escolar: Las ciencias biológicas y la historia. Discurso y Sociedad, 4(2), 294-331.

Moss, G., Barletta, N., Chamorro, D. \& Mizuno, J. (2013) La metáfora gramatical en los textos escolares de Ciencias Sociales en español. Onomažin, 28, 88-104.

Oteíza, T. (2006). El discurso pedagógico de la historia. Un análisis lingüistico sobre la construcción ideológica de la historia de Chile (1970-2001). Santiago: Frasis Editores.

Oteíza, T. (2009). Solidaridad ideológica en el discurso de la historia: Tensión entre orientaciones monoglósicas y heteroglósicas. Revista Signos. Estudios de Lingüística, 42(70), 219-244.

Oteíza, T. (2011). Representación de las memorias del pasado: Intersubjetividad en el discurso pedagógico de la historia. En T. Oteíza \& D. Pinto (Eds.), En (re)construcción: Discurso, identidad y nación en los manuales escolares de historia y de ciencias sociales. (pp. 129-172). Santiago: Editorial Cuarto Propio.

Oteíza, T. \& Pinuer, C. (2012). Prosodia valorativa: Construcción de eventos y procesos en el discurso de la historia. Discurso \& Sociedad, 6(2), 418-446.

Oteíza, T., Dalla Porta, C. \& Garrido, M. (2014). La evidencialidad en la construcción de la significación histórica por estudiantes de Licenciatura de Historia. Onomáz̨in. Número Especial IX ALSFAL, 57-80.

Oteíza, T., Henríquez, R. \& Pinuer, C. (2015). History class interactions and the transmission of recent Chilean memory of human rights violations. Journal of Educational Media, Memory and Society, 7(2) 44-67.

Peck, C. \& Seixas, P. (2008). Benchmarks of historical thinking: First steps. Canadian Journal of Education, 31(4), 1015-1038. 
Wineburg, S. (1991). Historical problem solving: A study of the cognitive processes used in the evaluation of documentary and pictorial evidence. Journal of Educational Psychology Journal of Educational Psychology, 83(1), 73-87.

\section{ANEXOS}

\section{Anexo 1: Texto completo de ID 24}

\begin{tabular}{|c|c|}
\hline Número de ejemplo en el texto & $\begin{array}{l}\text { Etapa y fase de } \\
\text { realización }\end{array}$ \\
\hline $\begin{array}{l}\text { Ejemplo 1. El texto nos ayuda a encajar la evolución del conocimiento europeo } \\
\text { sobre la geografía y su expansión. }\end{array}$ & $\begin{array}{l}\text { Inicio. Fase } \\
\text { ausencia de } \\
\text { propósito }\end{array}$ \\
\hline $\begin{array}{l}\text { Ejemplo 5. Este proceso de evolución fue muy largo y comprendió los siglos XI- } \\
\text { XVIII. }\end{array}$ & $\begin{array}{l}\text { Contexto. Fase } \\
\text { de inclusión de } \\
\text { tiempo y/o } \\
\text { espacio }\end{array}$ \\
\hline $\begin{array}{l}\text { Ejemplo 8. La primera fuente (mundo según los ingleses, siglo XI) nos muestra la } \\
\text { visión que tenían los europeos de la edad media. Debido a las características del } \\
\text { mundo medieval, este mapa presenta un mundo teocéntrico. La segunda fuente } \\
\text { (cantino, Portugal, siglo XVI) este mapa presenta una visión de mundo } \\
\text { directamente relacionada con el motivo de los descubrimientos geográficos, en la } \\
\text { cartografía por la aparición de los puntos cardinales. La tercera fuente (mapamundi } \\
\text { de Deslile, siglo XVIII) el conocimiento científico se hace cada vez más preciso, y } \\
\text { en los conocimientos geográficos aparecen los polos. }\end{array}$ & $\begin{array}{l}\text { Reformulación. } \\
\text { Fase de Crónica } \\
\text { orientada a } \\
\text { consecuencias }\end{array}$ \\
\hline $\begin{array}{l}\text { Ejemplo 11. El conocimiento permitió la expansión del mundo europeo, ya que } \\
\text { el conocimiento se tradujo en mejores representaciones del mapamundi el cual } \\
\text { permitió la mejor navegación y ubicación en el planeta para la expansión europea }\end{array}$ & $\begin{array}{l}\text { Cierre. Fase de } \\
\text { orientación por } \\
\text { interpretación }\end{array}$ \\
\hline
\end{tabular}

\section{Anexo 2: Figura 1. Fases y etapas del género 'recuento histórico'}

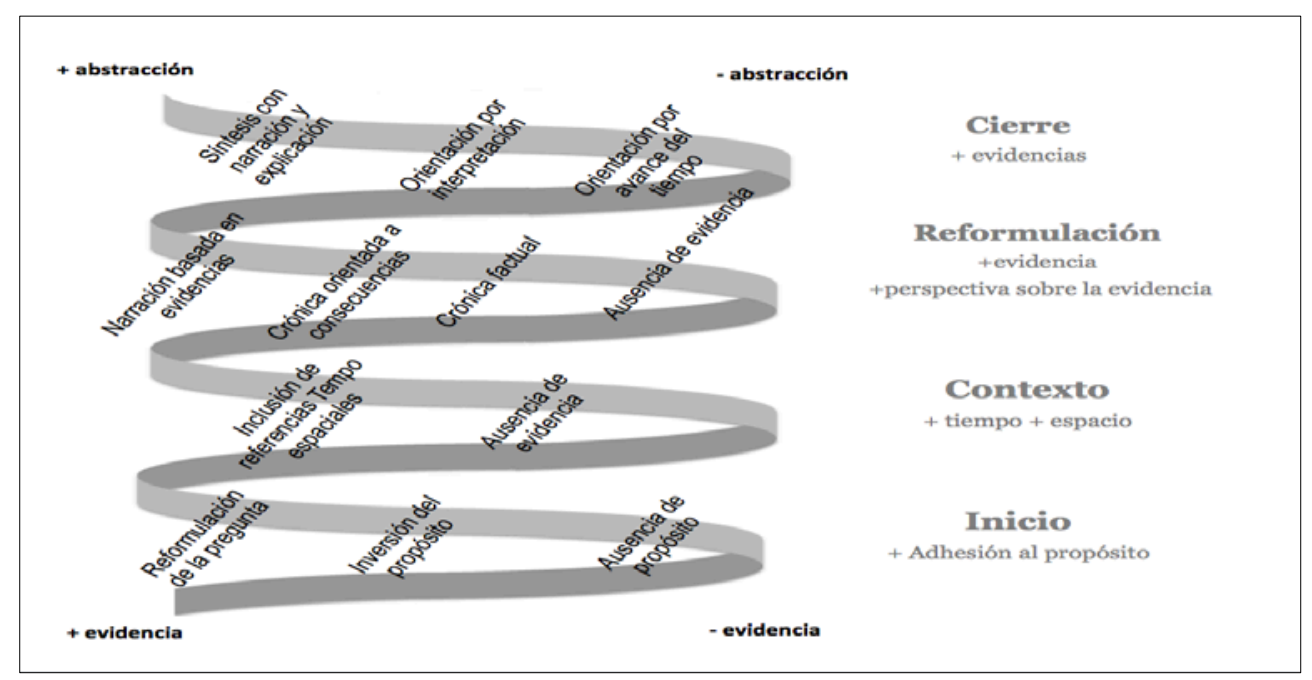

